\title{
Expansion of a compressible non-barotropic fluid in vacuum
}

\author{
Rongfeng $\mathrm{Yu}^{1}$ \\ ${ }^{1}$ Sun Yat-Sen University
}

September 17, 2020

\begin{abstract}
In this paper, we consider a region occupied by viscous or inviscid compressible magnetohydrodynamic fluids, and surrounded by vacuum. It is shown that the fluid region will expand at least linearly in time as soon as there are no singularities. The expanding rate is proportional to initial total energy and is inversely proportional to initial mass. The result indicates an interesting fact that the expansion of the viscous monatomic fluids seems similar to the inviscid fluids.
\end{abstract}

\section{Hosted file}

Expansion_of_compressible_nonbarotropic_fluid.pdf available at https://authorea.com/users/ 359770/articles/481579-expansion-of-a-compressible-non-barotropic-fluid-in-vacuum 